\title{
Estimation of mechanical properties of single wall carbon nanotubes using molecular mechanics approach
}

\author{
P SUBBA RAO $^{1,2, *}$, SUNIL ANANDATHEERTHA ${ }^{3}$, \\ G NARAYANA NAIK $^{1}$ and S GOPALAKRISHNAN ${ }^{1}$ \\ ${ }^{1}$ Department of Aerospace Engineering, Indian Institute of Science, \\ Bangalore 560012, India \\ ${ }^{2}$ Structures group, ISRO Satellite Centre, Bangalore 560017, India \\ ${ }^{3}$ Department of Mechanical Engineering, PES University, Bangalore 560085, India \\ e-mail: subbarao@isac.gov.in; subbarao_pendyala@yahoo.com
}

MS received 26 September 2012; revised 4 January 2015; accepted 11 February 2015

\begin{abstract}
Molecular mechanics based finite element analysis is adopted in the current work to evaluate the mechanical properties of Zigzag, Armchair and Chiral Single wall Carbon Nanotubes (SWCNT) of different diameters and chiralities. Three different types of atomic bonds, that is Carbon-Carbon covalent bond and two types of Carbon-Carbon van der Waals bonds are considered in the carbon nanotube system. The stiffness values of these bonds are calculated using the molecular potentials, namely Morse potential function and Lennard-Jones interaction potential function respectively and these stiffness's are assigned to spring elements in the finite element model of the CNT. The geometry of CNT is built using a macro that is developed for the finite element analysis software. The finite element model of the CNT is constructed, appropriate boundary conditions are applied and the behavior of mechanical properties of CNT is studied.
\end{abstract}

Keywords. Molecular mechanics; single wall carbon nanotube; mechanical properties; finite element method.

\section{Introduction}

Carbon nanotubes regarded as one of the most exciting nanomaterials with exceptional mechanical, physical, thermal and electronic properties are broadly classified into two types, Single Walled Carbon Nanotubes (SWCNTs) and Multi Walled Carbon Nanotubes (MWCNTs). There are three well-known configurations of SWCNTs namely Armchair, Zigzag and Chiral (Hamada et al 1992). Many works have been carried out regarding the estimation of their mechanical properties by various methods. Mielke et al (2004) employed density

${ }^{*}$ For correspondence 
functional theory (DFT) calculations and estimated the tensile strength and maximum tensile strain as $110 \mathrm{GPa}$ and $30 \%$ for $(5,5)$ Armchair SWCNT and $105 \mathrm{GPa}$ and $20 \%$ for $(10,0)$ Zigzag SWCNT. Ogata \& Shibutani (2003) also used the same method and estimated the tensile strength and maximum tensile strain as $115 \mathrm{GPa}$ and $29.5 \%$ for $(8,8)$ Armchair SWCNT and $107 \mathrm{GPa}$ and $20.8 \%$ for $(10,0)$ Zigzag SWCNT. Liew et al (2004) used molecular dynamics (MD) method and estimated that their values to be $140.4 \mathrm{GPa}$ and $28 \%$ for $(10,10)$ SWCNT and $110 \mathrm{GPa}$ and $20 \%$ for $(12,12)$ SWCNT. Xiao et al (2005) developed an analytical molecular structural mechanics model and predicted the tensile strengths of $126.2 \mathrm{GPa}$ for Armchair nanotubes and 94.5 GPa for Zigzag nanotubes. The failure strain for Armchair nanotubes (23.1\%) is found to be greater than that of Zigzag nanotubes (15.6-17.5\%) in their analysis. Natsuki \& Endo (2004) presented a structural mechanics approach based on continuum shell model and evaluated the tensile strength of Armchair $(10,10)$ and Zigzag $(17,0)$ nanotubes as $90 \mathrm{GPa}$ and $67 \mathrm{GPa}$ respectively. By thermally inducing the vibration of cantilevered MWCNT, Treacy et al (1996) measured the Young's modulus of carbon nanotubes in the range of 0.4-4.15 TPa. Krishnan et al (1998) used the same method to calculate the Young's modulus of SWCNTs and obtained an averaged value of 1.3 TPa. Mielke et al (2004) using DFT calculations found a Young's modulus of $0.96 \mathrm{TPa}$ for $(5,5)$ SWCNT and $0.94 \mathrm{TPa}$ for $(10,0)$ SWCNT. Jin \& Yuan (2003) used molecular dynamics (MD) simulations and calculated the Young's modulus for Armchair SWCNTs as $1.350 \mathrm{TPa}$ and 1.238 TPa. Meo \& Rossi (2006a) (Composites Science and Technology) used molecular mechanics (MM) based finite element analysis and predict a Young's modulus of $0.920 \mathrm{TPa}$ for Armchair nanotubes, 0.912 TPa for Zigzag nanotubes and 0.915 TPa for chiral nanotubes.

In this paper, we have considered the effect of non-bonded Lennard-Jones atomic interactions (van der Waals interactions) as well, which has been neglected in previous research works (Odegard et al 2002; Chang \& Gao 2003; Xiao 2005; Meo \& Rossi 2006b (Engineering Fracture Mechanics); Rossi \& Meo 2009). Furthermore, the work carried out by Natsuki \& Endo (2004), Xiao et al (2005) and Sun \& Zhao (2005) in the direction of evaluation of CNT mechanical properties, such as ultimate tensile strength, have not considered any effects of van der Waals interactions in their simulations. In this paper, we have paid due consideration to the effect of these non-bonded interactions.

This paper is organized as follows. Following the introduction in section 1, section 2 discusses the methodology of basic molecular mechanics adopted in this work as applied to modeling the carbon-carbon bonded interactions of carbon nanotube. Here, the details of Morse potential and Lennard-Jones potential functions are presented. This is followed by section 3 , highlighting the adoption of molecular mechanics method to finite element analysis and some of the novelties of molecular mechanics based finite element analysis. This is followed by section 4 on numerical simulation, where the method used to calculate the Young's modulus and tensile strength is discussed. The obtained results are presented and discussed in section 5 followed by conclusions in section 6 .

\section{Molecular mechanics methodology}

Molecular mechanics based finite element analysis is adopted in the current work to evaluate the above-mentioned mechanical properties of Zigzag, Armchair and Chiral SWCNTs of different diameters and chiralities.

Firstly, the geometry of SWCNT is built using the macro that is developed for the finite element analysis software named ANSYS. Later, three different types of atomic bonds are 
identified in the carbon nanotube system. They are Carbon-Carbon Covalent bond and two types of Carbon-Carbon van der Waals bonds. The two latter types of bonding are differentiated by the difference in the inter-atomic separation distance. Note that, such an interaction has never been considered by previous researchers (Odegard et al 2002; Chang \& Gao 2003; Xiao et al 2005; Meo \& Rossi 2007) in their molecular mechanics based finite element model.

In the finite element analysis, spring elements represent the covalent and the van der Waals bonding. The stiffness values of these elements are calculated using the molecular potentials, namely Morse potential function and Lennard-Jones 6-12 interaction potential function, the details of which are explained. Once the Finite Element model of the SWCNT is constructed, appropriate boundary conditions are applied and the mechanical properties of SWCNT are calculated.

\subsection{Molecular mechanics model}

Figure 1(a) shows two carbon atoms separated by a distance $r$. While one of the atoms is constrained against deformation, the other is subjected to an axial stretch, $\Delta r$ as shown in figure 1(a). $F_{\Delta \mathrm{r}}$ is the reaction force generated due to the imposed stretch deformation and $K_{\Delta \mathrm{r}}$ is the axial stiffness of the bond opposing the deformation. Figure 1(b) shows a system of three carbon atoms contained in a CNT. Under deformation of CNT, atoms C1, C2 and C3 move out of their equilibrium positions by $\left(\Delta X_{C 1}, \Delta Y_{C 1}, \Delta Z_{C 1}\right),\left(\Delta X_{C 2}, \Delta Y_{C 2}, \Delta Z_{C 2}\right)$ and $\left(\Delta X_{C 3}, \Delta Y_{C 3}, \Delta Z_{C 3}\right)$ respectively. This results in a change in the bond angle $\Delta \theta$ between $\mathrm{C} 1-\mathrm{C} 2$ and $\mathrm{C} 1-\mathrm{C} 3$ covalent bonds. This $\Delta \theta$ results in a change in the bond-angle potential, $V_{\Delta \theta}$. The modified Morse potential $V_{M P}$ is written as (Belytschko et al 2002),

$$
V_{M P}=V_{\text {stretch }}+V_{\text {angle }}=V_{\Delta R}+V_{\Delta \theta}
$$

where $V_{\Delta R}$ (i.e. $V_{\text {stretch }}$ ) and $V_{\Delta \theta}$ (i.e. $V_{\text {angle }}$ ) are bond stretch and bond angle potentials respectively.

i) Before stretching.

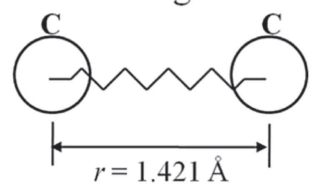

ii ) After stretching.

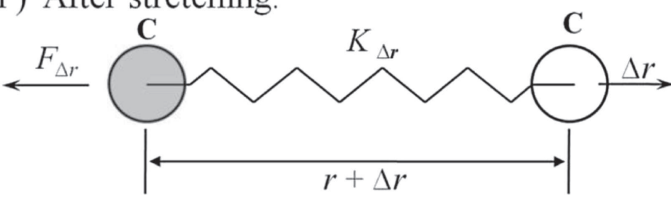

(a)

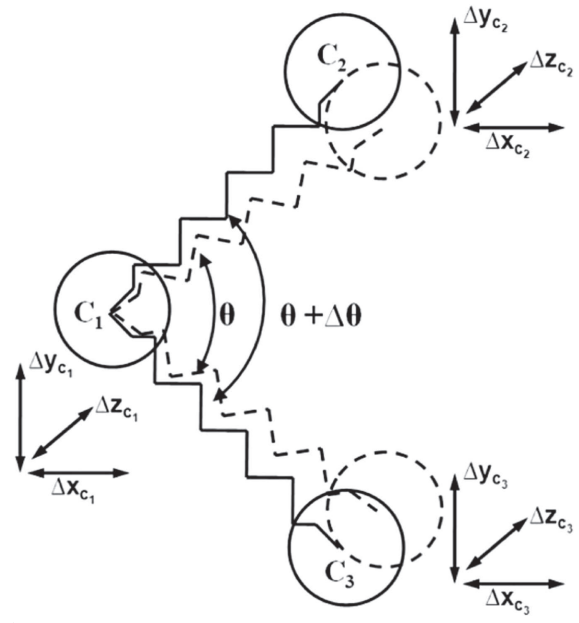

(b)

Figure 1. (a) Spring elements representing bond - stretching and (b) bond bending. 
The bond stretch potential is given by the relation (Belytschko et al 2002),

$$
V_{\Delta r}=D_{e}\left[\left(1-e^{-\beta \cdot \Delta r}\right)^{2}-1\right]
$$

where ' $D_{e}$ ' is the equilibrium binding energy, ' $\beta$ ' is the fitting parameter and ' $\Delta r$ ' is $\left(r-r_{o}\right)$, where $r_{o}$ is the equilibrium distance of separation between the two carbon atoms in the CNT $\left(r_{o}=0.1421 \mathrm{~nm}\right)$ and ' $r$ ' is the distance of separation between the two carbon atoms after stretch. This $\Delta r$ results in a change in the bond potential function $V_{\Delta r}$ given by Eq. (2). The values of these constants are $D_{e}=6.03105 \times 10^{-10} \mathrm{~N} \mathrm{~nm}, \beta=2.625 \times 10^{10} \mathrm{~m}^{-1}$ (Belytschko et al 2002).

The bond angle potential is given by the relation (Belytschko et al 2002; Meo \& Rossi 2006a)

$$
V_{\Delta \theta}=0.5 k_{\theta} \Delta \theta^{2}\left(1+k_{\text {sextic }}\left(\Delta \theta^{4}\right)\right)
$$

where $\Delta \theta$, i.e. $\left(\theta-\theta_{0}\right)$ is the variation in $\mathrm{C}-\mathrm{C}-\mathrm{C}$ bond angle (see figure $1(\mathrm{~b})$ ); $\theta$ is the angle of separation between the two bonds after deformation and $\theta_{0}$ is the equilibrium bond angle of separation between the two bonds. $k_{\theta}$ is the bond stiffness for angular deformations $\left(k_{\theta}=\right.$ $0.9 \mathrm{nN}-\mathrm{nm} / \mathrm{rad}^{2}$ ). The value of the constant $k_{\text {sextic }}$ in Eq. (3) was provided by Belytschko et al (2002) as $0.745 \mathrm{rad}^{-4}$

The Lennard-Jones 6-12 potential function (Lau et al 2004) is written as

$$
U_{i j}=4 \epsilon\left[\left(\frac{r_{c}}{r_{i j}}\right)^{12}-\left(\frac{r_{c}}{r_{i j}}\right)^{6}\right]
$$

where $U_{i j}$ is the van der Waals interaction potential between the $i^{\text {th }}$ and $j^{\text {th }}$ atoms of a system of two carbon atoms, separated by a distance of $r_{i j} . \epsilon$ is the Lennard-Jones parameter, $(\epsilon=$ $3.825 \times 10^{-4} \mathrm{nN}-\mathrm{nm}$ ) (Brcic et al 2009), whose value determines the strength of Lennard-Jones interaction. $r_{c}$ is the cut-off distance, which is equal to $0.34 \mathrm{~nm}$ for calculating the Lennard-Jones interactions.

\section{Molecular mechanics based finite element modeling}

In the present work, two types of finite elements are used to represent the covalent and van der Waals inter-atomic interactions respectively. They are chemical bond element and Lennard-Jones element.

\subsection{Novelties of molecular mechanics based finite element approach}

1. The finite elements are governed by structural equivalents of molecular potential functions; thereby the inter-atomic interactions are accurately depicted.

2. Since the nanoscale inter-atomic interactions are one to one, a single finite element can represent the individual interactions. Thus, the total number of finite elements in the nanoscale structure would be the same as the total number of nanoscale inter-atomic interactions, which eliminates the discretization errors.

3. Very large domains of the nanoscale structure can be analyzed at a fraction of the computational time and resources as opposed to methods such as molecular dynamics and density functional theory. 


\subsection{Calculation of covalent bond stiffness}

First, to obtain the bond stiffness of the chemical bond element, Eq. (2) has to be differentiated twice with respect to $\Delta r$. Differentiating $V_{\Delta r}$ in Eq. (2) with respect to $\Delta r$,

$$
F_{\Delta r}=\frac{d V_{\Delta r}}{d r}=2 \beta D_{e} e^{-\beta \Delta r}\left(1-e^{-\beta \Delta r}\right)
$$

where $F_{\Delta r}$ is the force generated by the $\mathrm{C}-\mathrm{C}$ covalent bond against the deformation $r$,

Next, differentiating $F_{\Delta r}$ with respect to $\Delta r$, we get the bond stiffness, $K_{\Delta r}$ of the chemical bond element.

$$
K_{\Delta r}=\frac{d F_{\Delta r}}{d r}=\frac{d^{2} V_{\Delta r}}{d r^{2}}=\beta^{2} D_{e} e^{-\beta \Delta r}\left(2 e^{-\beta \Delta r}-1\right)
$$

For obtaining the bond bending stiffness, it is required to differentiate Eq. (3) twice with respect to $\Delta \theta$. Differentiating $V_{\Delta \theta}$, we get $M_{\Delta \theta}$, moment function,

$$
M_{\Delta \theta}=\frac{d V_{\Delta \theta}}{d \Delta \theta}=k_{\theta} \Delta \theta\left(1+3 k_{\text {sextic }} \Delta \theta^{4}\right)
$$

Next, differentiating $M_{\Delta \theta}$ we get, $K_{\Delta \theta}$ the bond bending stiffness with varying bond angle $\Delta \theta$. It is written as

$$
K_{\Delta \theta}=\frac{d M_{\Delta \theta}}{d \Delta \theta}=k_{\theta}\left(1+15 k_{\text {sextic }} \Delta \theta^{5}\right)
$$

\subsection{Calculation of Lennard-Jones stiffness}

Differentiating Uij in Eq. (4) with respect to $r_{i j}$, we get the Lennard-Jones force of interaction.

$$
\frac{d U_{i j}}{d r_{i j}}=F_{r_{i j}}=24 \frac{\epsilon}{r_{c}}\left[-2\left(\frac{r_{c}}{r_{i j}}\right)^{13}+\left(\frac{r_{c}}{r_{i j}}\right)^{7}\right]
$$

Differentiating $F_{r_{i j}}$ with respect to $r_{i j}$, we get the Lennard-Jones element stiffness

$$
\frac{d^{2} U_{i j}}{d r_{i j}^{2}}=\frac{d F_{i j}}{d r_{i j}}=K_{r_{i j}}=24 \frac{\epsilon}{r_{c}^{2}}\left[26\left(\frac{r_{c}}{r_{i j}}\right)^{14}-7\left(\frac{r_{c}}{r_{i j}}\right)^{8}\right]
$$

Figures 2(a) and (b) show the finite spring elements used in the present analysis. Two types of Lennard-Jones finite elements viz. Type 1 (LJSE1) and Type 2 (LJSE2) of lengths $0.2461 \mathrm{~nm}$ and $0.2842 \mathrm{~nm}$ respectively are used. The respective stiffness values calculated using Eq. (10) for the above-mentioned lengths are $183.1685 \mathrm{nN} / \mathrm{nm}$ and $23.067 \mathrm{nN} / \mathrm{nm}$ respectively. Similarly, at deformed covalent bond lengths of $0.1421 \mathrm{~nm}$ (that is, at $\Delta r \rightarrow 0$ ) it can be seen from Eq. 6, that the bond stiffness is $831.4514 \mathrm{nN} / \mathrm{nm}$. From the above discussion, it is concluded that the stiffness of LJSE1 and LJSE2 is not negligible in comparison to the stiffness of the C-C covalent bond and hence are considered in the present study. The relation between the element load vector $\{\mathrm{Q}\}$, the element stiffness matrix $[\mathrm{K}]$ and the element displacement vector $\{\mathrm{q}\}$ is written as $\{\mathrm{Q}\}=[\mathrm{K}]\{\mathrm{q}\}$. 


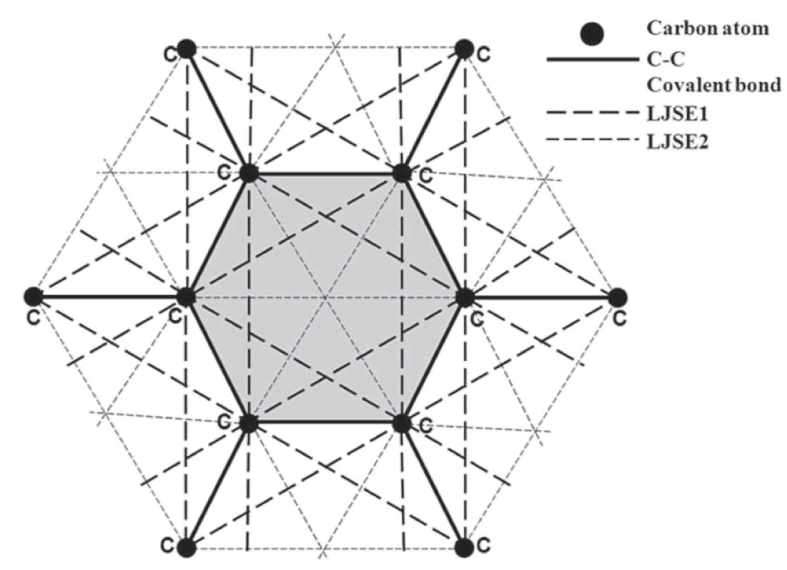

(a)

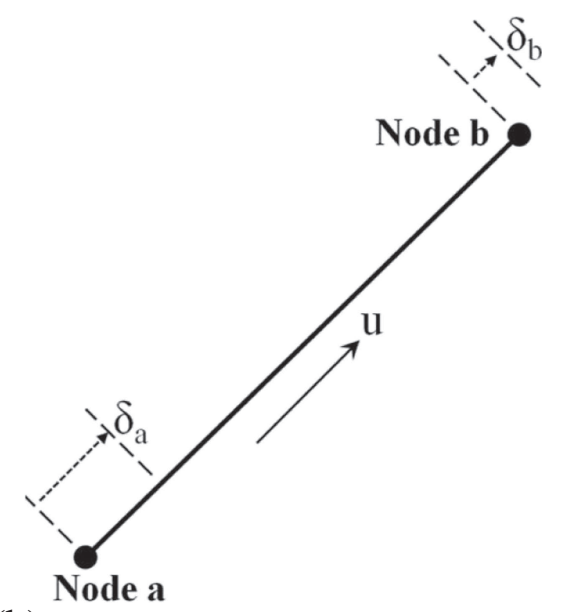

(b)

Figure 2. (a) Lennard-Jones Spring Elements-Type 1 (LJSE1) $\left(r_{i j}=\sqrt{3} L_{C-C}=0.2461 \mathrm{~nm}\right)$ and Type 2 (LJSE2) $\left(r_{i j}=2 L_{C-C}=0.2842 \mathrm{~nm}\right) . L_{C-C}=0.1421 \mathrm{~nm}$. The continuous thick lines represent chemical bonds modeled by Eq. (6). Dashed thick lines represent LJSE1 while dashed thin lines represent LJSE2; both modeled by Eq. (10). (b) 3D extensional spring element in its local co-ordinate system.

\section{Numerical simulation}

For numerical simulation studies, SWCNTs of different chirality and diameters are considered. ANSYS software is used for the finite element simulations. Geometry of the SWCNT is constructed using the macro written in software's Parametric DesignLanguage (APDL). Finite elements COMBIN39 and COMBIN14 are chosen for analysis. COMBIN39 is a nonlinear spring element defined with the help of force deflection behavior obtained using Eq. 6 . COMBIN14 is a linear spring-damper element and is employed with its damping coefficient value set to zero. The stiffness values of the springs are calculated using Eqs. (6) and (10). Molecular Mechanics based finite element analysis is carried out to study the effect of chirality and diameter on the tensile strength, maximum tensile strain and Young's modulus.

Figure 3(a) shows atomic bonding in the finite element model of $(20,20)$ Armchair SWCNT containing two unit cells and figure 3 (b) shows the finite element model of $(6,6)$ carbon nanotube. The edges of the regular hexagons represent the $\mathrm{C}-\mathrm{C}$ covalent bonds while the shorter and longer diagonals of each of the regular hexagons represent LJSE1 and LJSE2 respectively.

\subsection{Longitudinal tensile behavior of SWCNTs}

In the molecular mechanics based finite element simulation of SWCNTs subjected to longitudinal loading, all atoms on one end are constrained and longitudinal tensile displacement is applied to all atoms on the opposite end. Initially, the geometry of the nanotube is built and the finite elements are defined. The ability of the software ANSYS10.0 to use only certain types of ForceDeflection (FD) types (to which the covalent FD behavior belongs and the Lennard-Jones FD behavior does not belong) is the reason behind the use of non-linear element to simulate Covalent bonds (COMBIN39) and linear spring to simulate Lennard-Jones interactions (COMBIN 14). Then, all atoms on one end of the SWCNT are constrained. 


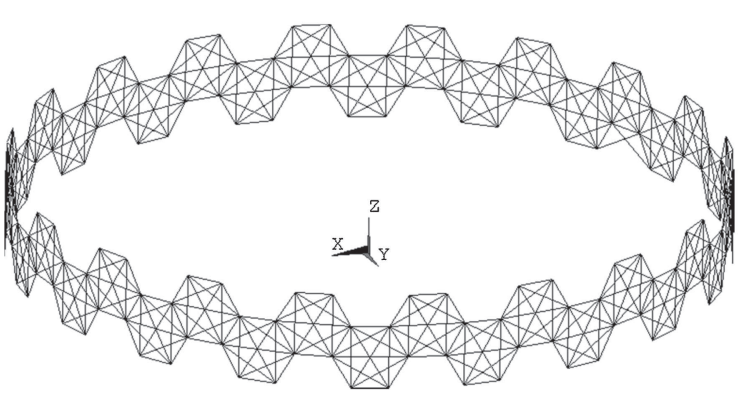

(a)

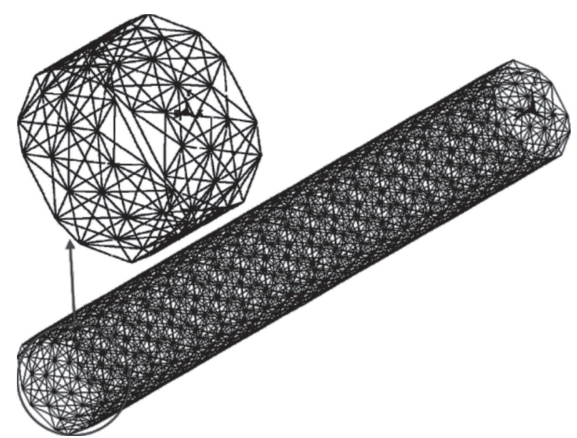

(b)

Figure 3. (a) Atomic bonding in the Finite Element Model of $(20,20)$ Armchair SWCNT Containing two unit cells; (b) Finite Element Model of $(6,6)$ CNT.

For simulation of tensile behavior, maximum tensile strain (that is, the strain at which the stress-strain curve for that particular nanotube just starts to dip) is applied to the SWCNT. This displacement is applied in suitable steps in the non-linear finite element analysis so as to obtain a smooth and well defined stress-strain behavior. Once, the above displacement boundary conditions are set, finite element analysis is performed. After the completion of analysis, the tensile stress is calculated as follows.

$$
\sigma_{s w c n t}^{\|}=\frac{\sum_{j=1}^{k} F_{j}}{\pi D t}
$$

where ' $\sigma$ ' is the stress, the superscript ' $\|$ ' corresponds to the SWCNT axial direction, $\sum_{j=1}^{k} F_{j}$ is the sum of axial reaction forces on all atoms $(\mathrm{j}=1$ to $\mathrm{k})$. The obtained stress is then plotted against the applied strain in each step of the analysis and the stress-strain behavior is obtained. The tensile stress at the point where the stress-strain curve just starts to dips is taken as the tensile

Table 1. Predicted and literature values of tensile strength and maximum tensile strain of SWCNT.

\begin{tabular}{lccccccr}
\hline $\begin{array}{l}\text { Different } \\
\text { chiralites }\end{array}$ & $(\mathrm{n}, \mathrm{m})$ & Diameter $(\mathrm{mm})$ & $\begin{array}{c}\text { Predicted } \\
\text { tensile } \\
\text { strength }\end{array}$ & $\begin{array}{c}\text { Predicted } \\
\text { tensile } \\
\text { strain }(\%)\end{array}$ & $\begin{array}{c}\text { Reported } \\
\text { literature } \mathrm{X}_{\mathrm{T}}\end{array}$ & $\begin{array}{c}\text { Reported } \\
\text { literature } \varepsilon_{\text {max }}\end{array}$ & Method \\
\hline Arm chair & $(5,5)$ & 0.6785 & 117.05 & 29.12 & $110.0^{1}$ & $30^{1}$ & DFT \\
& $(7,7)$ & 0.9499 & 118.88 & 29.62 & $121.3^{2}$ & $20.68^{2}$ & MM \\
& $(9,9)$ & 1.2213 & 120.45 & 29.69 & & & \\
Average & $(11,11)$ & 1.4927 & 122.53 & 29.98 & & & \\
Zigzag & $(5,0)$ & 0.3917 & 119.73 & 29.60 & & & \\
& $(8,0)$ & 0.6267 & 101.21 & 20.56 & $105^{1}$ & & \\
& $(10,0)$ & 0.7834 & 102.69 & 20.64 & & & \\
Average & $(12,0)$ & 0.9401 & 103.23 & 20.75 & & & \\
Chiral & $(5,4)$ & 0.6119 & 114.22 & 22.15 & & & \\
Average & $(10,1)$ & 0.8254 & 117.96 & 22.98 & & & \\
\hline
\end{tabular}

${ }^{1}$ Mielke et al (2004), ${ }^{2}$ Meo \& Rossi (2007). 
strength of the SWCNT. SWCNT geometric parameters - ' $t$ ' is the thickness of the SWCNT and ' $\mathrm{D}$ ' is the SWCNT diameter.

\subsection{Calculation of Young's modulus of SWCNTs}

As explained above, the stress-strain behaviors of various SWCNTs are calculated first. Then, from these stress-strain diagrams, the value of Young's modulus is calculated as the slope of the linear region of the curve (the initial region of the curves is taken to be the linear region of the curve).
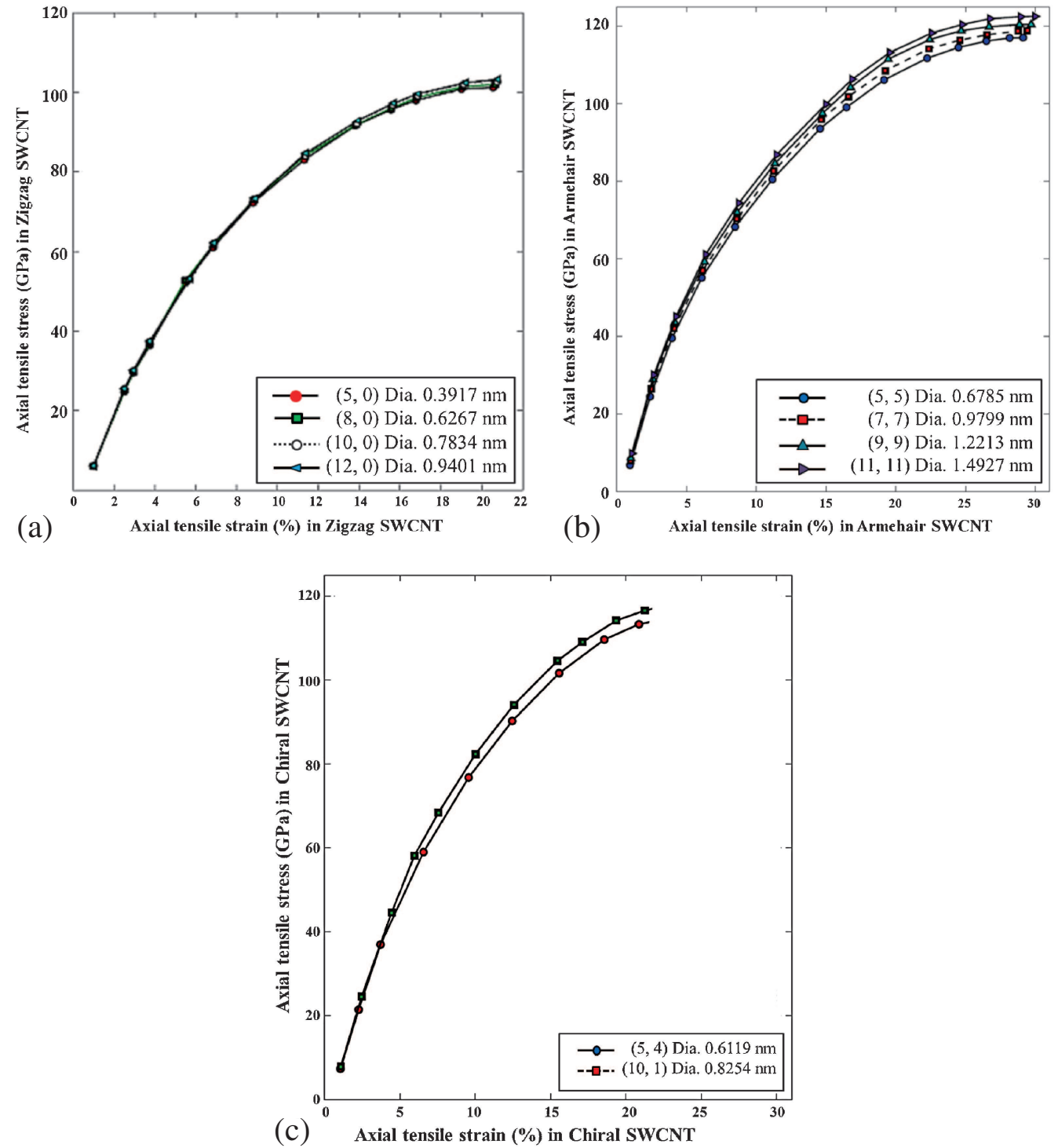

Figure 4. (a) Tensile stress strain behavior of Zigzag SWCNT; (b) Tensile stress strain behavior Armchair SWCNT; (c) Tensile stress strain behavior of Chiral SWCNT. 


\section{Results and discussions}

The tensile behavior of SWCNTs of Zigzag, Armchair and Chiral configurations of different diameter and chiralities is studied. The longitudinal tensile Young's modulus and maximum tensile strength are calculated and the results obtained are compared with published literature data.

\subsection{Longitudinal tensile behavior}

Table 1 shows the values of longitudinal tensile strength and maximum failure strain for various SWCNTs obtained using the present method, wherein, the Lennard-Jones inter-atomic interactions are also considered. The table also provides a comparison of the obtained values with those of published literature. From the comparison, it can be seen that the obtained values of longitudinal tensile strength and maximum tensile strain are in very good agreement with the published literature values.

Figure 4(a), (b) and (c) respectively shows the tensile behavior of Zigzag, Armchair and Chiral SWCNTs. Tensile strength and maximum tensile strain of Armchair SWCNTs are greater than that of Zigzag and Chiral SWCNTs as reported in numerous published literatures (Mielke et al 2004; Ogata et al 2003; Meo \& Rossi 2007).

\subsection{Estimation of Young's modulus}

Young's modulus of Zigzag, Armchair and Chiral SWCNTs is calculated using the slope of the stress strain diagram. The obtained values of Young's modulus are provided in table 2. It can be seen from table 2 that, the Young's modulus of Zigzag SWCNTs are greater than that of Armchair and Chiral SWCNTs. It can also be seen that its value is sensitive to the SWCNT diameter.

Table 2. Predicted SWCNT Young's modulus.

\begin{tabular}{lccccc}
\hline $\begin{array}{l}\text { Different } \\
\text { chiralites }\end{array}$ & $(\mathrm{n}, \mathrm{m})$ & $\begin{array}{c}\text { Diameter } \\
(\mathrm{nm})\end{array}$ & $\begin{array}{c}\text { Predicted Young's } \\
\text { modulus } E^{\|}(\mathrm{TPa})\end{array}$ & $\begin{array}{c}\text { Reported } \\
\text { literature } E^{\|}(\mathrm{TPa})\end{array}$ & Method \\
\hline Armchair & $(5,5)$ & 0.6785 & 1.2289 & $1.28-1.48^{1}$ & MD \\
& $(7,7)$ & 0.9499 & 1.2678 & $1.293^{2}$ & DFT \\
& $(9,9)$ & 1.2213 & 1.2767 & $0.92^{3}$ & MM \\
& $(11,11)$ & 1.4927 & 1.2811 & $1.01^{4}$ & DFT \\
Average & & & & $1.320^{2}$ & DFT \\
Zigzag & $(5,0)$ & 0.3917 & 1.2636 & & \\
& $(8,0)$ & 0.6267 & 1.2436 & $1.912^{3}$ & MM \\
& $(10,0)$ & 0.7834 & 1.2778 & $1.6^{5}$ & DFT \\
Average & $(12,0)$ & 0.9401 & 1.2895 & & DFT \\
Chiral & $(5,4)$ & 0.6119 & 1.2688 & $0.915^{3}$ & MM \\
Average & $(10,1)$ & 0.8254 & 1.1627 & & \\
\hline
\end{tabular}

${ }^{1}$ Wang et al (2005), ${ }^{2}$ Peng et al (2006), ${ }^{3}$ Meo \& Rossi (2007), ${ }^{4}$ Treacy et al (1996) and ${ }^{5}$ Mielke et al (2004). 


\section{Conclusions}

Molecular mechanics based finite element analysis is carried out for all three chiralities of SWCNT (armchair, zigzag and chiral) of different diameters. Mechanical properties such as tensile strength and Young's modulus are calculated using SWCNT tensile behavior. The effect of non-bonded Lennard-Jones interactions is also considered for study. The effect of inclusion of these non-bonded interactions is studied by making the numerical stiffness of these bonds to negligible levels and found that they significantly reduce the mechanical properties. Thus, the importance of inclusion of Lennard-Jones interactions is highlighted in this paper. All further calculations are carried out by considering the full effect of these non-bonded interactions. It is found that the longitudinal tensile strength and maximum tensile strain of Armchair SWCNTs is greater than that of Zigzag and Chiral SWCNTs and its value increases with increasing SWCNT diameter. The average value of longitudinal tensile strength of Armchair SWCNTs is found to be $17.07 \%$ and $3.13 \%$ greater than that of Zigzag and Chiral SWCNTs. The average value of longitudinal tensile strength of Armchair, Zigzag and Chiral SWCNTs is 119.73, 102.27 and 116.09 GPa respectively. The average value of maximum tensile strain of Armchair SWCNTs is found to be $43.16 \%$ and $31.18 \%$ greater than that of Zigzag and Chiral SWCNTs. Its average values for Armchair, Zigzag and Chiral SWCNTs correspond to 29.6\%, 20.6\% and 22.5\% respectively. The longitudinal Young's modulus of SWCNTs is calculated as the slope of the linear region of the stress-strain diagram. Its averaged values for Armchair, Zigzag and Chiral SWCNTs are 1.26, 1.26 and 1.18 TPa respectively. The calculated values of the mechanical properties listed above agree well with the published literature data.

\section{Acknowledgements}

The authors thank the Structures group, ISRO Satellite Centre and Department of Aerospace Engineering, Indian Institute of Science for allowing us to make use of the necessary facilities for carrying out this research work. Authors also thank the reviewers and the editor for the valuable inputs and suggestions.

\section{References}

Brcic M, Canadija M, Brnic J, Lanc D, Krscanski S and Vukelic G 2009 FE Modelling of multi-walled carbon nanotubes. Estonian J. Eng. 15(2): 77-86

Belytschko T, Xiao S P, Schatz G C and Ruoff R S 2002 Atomistic simulations of nanotube fracture. Phys. Rev. B 65: 235430:1-8

Chang T and Gao H 2003 Size dependent elastic properties of a single carbon nanotube via molecular mechanics. J. Mech. Phys. Solids 51: 1059-74

Hamada N, Sawada S and Oshiyama A 1992 New one dimensional conductors: Graphitic micro tubes. Phys. Rev. B 68: 1579

Jin Y and Yuan F G 2003 Simulation of elastic properties of single walled carbon nanotubes. Composites Sci. Technol. 63: 1507-1515

Krishnan A, Dujardin E, Ebbesen T W, Yianilos P N and Treacy M M J 1998 Young's modulus of single walled carbon nanotubes. Phys. Rev. B 58(20): 14013-14019

Lau K T, Chipara M, Ling H Y and Hui D 2004 On the effective elastic moduli of carbon nanotubes for nanocomposite structures. Composites B: Eng. Nanocomposites 35(2): 95-101

Liew K M, He X Q and Wong C H 2004 On the study of elastic and plastic properties of multi-walled carbon nanotubes under axial tension using molecular dynamics simulation. Acta Mater. 52: 2521-2527 
Meo M and Rossi M 2006a Prediction of Young's modulus of single wall carbon nanotubes by molecular - mechanics based finite element modeling. Composites Sci. Technol. 66: 1597-1605

Meo M and Rossi M 2006b Tensile failure prediction of single wall carbon nanotube. Eng. Fracture Mech. 73: 2589-2599

Meo M and Rossi M 2007 A molecular-mechanics based finite element model for strength prediction of single wall carbon nanotubes. Mater. Sci. Eng. A 454-455: 170-177

Mielke S L, Troy D, Zhang S, Li J L, Xiao S, Car R, Ruoff R S, Schatz G C and Belytschko T 2004 The role of vacancy defects and holes in the fracture of carbon nanotubes. Chem. Phys. Lett. 390: 413-420

Natsuki T and Endo M 2004 Stress simulation of carbon nanotubes in tension and compression. Carbon 42: $2147-2151$

Odegard G M, Gates T S, Nicholson L M and Wise K E 2002 Equivalent-continuum modelling of nano structured materials. Composites Sci. Technol. 62: 1869-80

Ogata S and Shibutani Y 2003 Ideal tensile strength and band gap of single-walled carbon nanotubes. Phys. Rev. B 68: 165409

Peng Y J, Zhang L Y, Jin Q H, Li B H and Ding D T 2006 Ab-initio studies of elastic properties and electronic structures of $\mathrm{C}$ and $\mathrm{BN}$ nanotubes. Physica E 33: 155-159

Rossi M and Meo M 2009 On the estimation of mechanical properties of single-walled carbon nanotubes by using a molecular-mechanics based FE approach. Composites Sci. Technol. 69: 1394-1398

Sun X and Zhao W 2005 Prediction of stiffness and strength of single-walled carbon nanotubesby molecular - mechanics based finite element approach. Mater. Sci. Eng. A 390: 366-371

Treacy M M L, Ebbesen T W and Gibson J M 1996 Exceptionally high Young's modulus observed for individual carbon nanotubes. Nature (London) 381: 678-680

Wang Y, Wang X, Ni X and Wu H 2005 Simulation of the elastic response and the buckling modes of single-walled carbon nanotubes. Comput. Mater. Sci. 32: 141-146

Xiao J R, Gama B A and Gillespie J W 2005 An analytical molecular structural mechanics model for the mechanical properties of carbon nanotubes. Int. J. Solids Struct. 42: 3075-3092 